\title{
Assessing genetic divergence in interspecific hybrids of Aechmea gomosepala and A. recurvata var. recurvata using inflorescence characteristics and sequence-related amplified polymorphism markers
}

\author{
F. Zhang, Y.Y. Ge, W.Y. Wang, X.L. Shen and X.Y. Yu \\ Flower Research and Development Centre, \\ Zhejiang Academy of Agricultural Sciences, Hangzhou, P.R. China \\ Corresponding author: F. Zhang \\ E-mail: zhangfei216@yahoo.com.cn
}

Genet. Mol. Res. 11 (4): 4169-4178 (2012)

Received March 5, 2012

Accepted June 22, 2012

Published September 27, 2012

DOI http://dx.doi.org/10.4238/2012.September.27.2

\begin{abstract}
Conventional hybridization and selection techniques have aided the development of new ornamental crop cultivars. However, little information is available on the genetic divergence of bromeliad hybrids. In the present study, we investigated the genetic variability in interspecific hybrids of Aechmea gomosepala and A. recurvata var. recurvata using inflorescence characteristics and sequencerelated amplified polymorphism (SRAP) markers. The morphological analysis showed that the putative hybrids were intermediate between both parental species with respect to inflorescence characteristics. The 16 SRAP primer combinations yield 265 bands, among which 154 $(57.72 \%)$ were polymorphic. The genetic similarity was an average of 0.59 and ranged from 0.21 to 0.87 , indicating moderate genetic divergence among the hybrids. The unweighted pair group method with arithmetic average (UPGMA)-based cluster analysis distinguished the
\end{abstract}


hybrids from their parents with a genetic distance coefficient of 0.54 . The cophenetic correlation was 0.93 , indicating a good fit between the dendrogram and the original distance matrix. The two-dimensional plot from the principal coordinate analysis showed that the hybrids were intermediately dispersed between both parents, corresponding to the results of the UPGMA cluster and the morphological analysis. These results suggest that SRAP markers could help to identify breeders, characterize $F_{1}$ hybrids of bromeliads at an early stage, and expedite genetic improvement of bromeliad cultivars.

Key words: Aechmea; Interspecific hybrid; Genetic divergence; SRAP

\section{INTRODUCTION}

Ornamental bromeliad (Bromeliaceae) is one of the most popular potted flowers worldwide. Aechmea constitutes an important component of bromeliad industry due to its varying flower color and type and increased cold resistance compared to other bromeliads (Neoregelia, Guzmania, and Vriesea). Thus, it is important to develop new Aechmea hybrids for enriching the bromeliad cultivars.

The conventional hybridization and selection system is the most efficient method of breeding new bromeliad varieties. The Florida Committee of Bromeliads Society (http://www. fcbc.org) reported that more than $90 \%$ of the ornamental bromeliad varieties are inter-specific hybrids, underscoring the importance of developing novel bromeliad hybrids in order to meet market demands. Therefore, elucidating and quantifying the genetic variability in a population is important for breeders to study the genetic relationships between hybrids and parents. This information could also guide the selection of promising materials and new crosses and elucidate the mechanism by which horticultural traits are inherited. Previous studies have assessed the genetic diversity among various bromeliad species (Izquierdo and Pinero, 2000; Barfuss et al., 2005; Sousa et al., 2008; Palma-Silva et al., 2009; Boisselier-Dubayle et al., 2010); however, little information is available on the genetic divergence among interspecific hybrids in Aechmea.

The sequence-related amplified polymorphism (SRAP) technique preferentially targets exon sequences and promoter regions (Li and Quiros, 2001). SRAP profiling has been used to assess genetic diversity (Han et al., 2008; Mishra et al., 2011), construct genetic maps (Zhang et al., 2011), and gene tagging (Li et al., 2003). These varied applications demonstrate its resolving capacity in genetic studies. The present study was designed to evaluate genetic variability among $\mathrm{F}_{1}$ hybrids derived from an interspecific cross between Aechmea gomosepala and $A$. recurvata var. recurvata.

\section{MATERIAL AND METHODS}

\section{Plant materials and DNA isolation}

Two Aechmea species, A. gomosepala and A. recurvata var. recurvata, were used to construct an interspecific hybrid population. The artificially controlled hybridization was car- 
ried out according to the method as described by Vervaeke et al. (2003). Both parents and the derived $\mathrm{F}_{1}$ hybrids were maintained at the Flower Research and Development Centre, Zhejiang Academy of Agricultural Sciences.

Young leaves were collected from each accession, frozen with liquid nitrogen, and ground into a powder. Genomic DNA was isolated following a cetyltrimethylammonium bromide (CTAB)-based procedure (Murray and Thompson, 1980). DNA concentration was estimated by comparison with known concentrations of lambda DNA on $0.8 \%$ agarose gels.

\section{SRAP profiling}

A total of 18 SRAP primer combinations of 6 forward primers and 3 reverse primers (Table 1) were initially used to screened for polymorphisms. As a result, 16 SRAP primer combinations were selected for their high level of polymorphism and clear banding patterns. All PCR amplifications were performed in a $10-\mu \mathrm{L}$ reaction volume containing $1 \mathrm{X}$ PCR buffer, $3 \mathrm{mM} \mathrm{Mg}^{2+}, 200 \mu \mathrm{M}$ dNTP, $0.5 \mathrm{U}$ Taq DNA polymerase (Takara, Dalian, China), $10 \mu \mathrm{M}$ of each primer, and $25 \mathrm{ng}$ DNA template. The amplification program was based on the report by $\mathrm{Li}$ and Quiros (2001) as follows: an initial denaturing step at $94^{\circ} \mathrm{C}$ for 5 min followed by 5 cycles of $94^{\circ} \mathrm{C}$ for $1 \mathrm{~min}, 35^{\circ} \mathrm{C}$ for $1 \mathrm{~min}$, and $72^{\circ} \mathrm{C}$ for $1 \mathrm{~min}$, followed by 35 cycles at $94^{\circ} \mathrm{C}$ for $1 \mathrm{~min}, 50^{\circ} \mathrm{C}$ for $1 \mathrm{~min}$, and $72^{\circ} \mathrm{C}$ for $1 \mathrm{~min}$, and a final extension step at $72^{\circ} \mathrm{C}$ for $7 \mathrm{~min}$. PCR products were electrophoresed on $8 \%$ non-denaturing polyacrylamide gels, run at $300 \mathrm{~V}$ for $2.5 \mathrm{~h}$ in $0.5 \mathrm{X}$ TBE buffer, and visualized by silver staining.

Table 1. Primer sequences for sequence-related amplified polymorphism analysis.

\begin{tabular}{|c|c|c|c|}
\hline Forward primer & Sequence $\left(5^{\prime} \rightarrow 3^{\prime}\right)$ & Reverse primer & Sequence $\left(5^{\prime} \rightarrow 3^{\prime}\right)$ \\
\hline $\mathrm{Me} 20$ & GAGTATCAACCCGGATT & Em1 & GACTGCGTACGAATTAAT \\
\hline Me21 & GTACATAGAACCGGAGT & Em2 & GACTGCGTACGAATTTGC \\
\hline \multirow[t]{4}{*}{$\mathrm{Me} 22$} & TACGACGAATCCGGACT & Em3 & GACTGCGTACGAATTGAC \\
\hline & & Em4 & GACTGCGTACGAATTTGA \\
\hline & & Em5 & GACTGCGTACGAATTAAC \\
\hline & & Em6 & GACTGCGTACGAATTGCA \\
\hline
\end{tabular}

\section{Data scoring and statistical analysis}

Amplified bands were scored as present or absent ( 1 or 0$)$ across the 2 parental species for each primer combination. For each primer combination, the total number of amplified bands (TB), the number of polymorphic bands (PB), the percentage of polymorphic bands (PPB), and polymorphic information content (PIC) were calculated by PowerMarker v3.25 (Liu and Muse, 2005). The resolving power (Rp) of a primer combination was estimated through a formula $\mathrm{Rp}=\Sigma \mathrm{Ib}$. Band informativeness $(\mathrm{Ib})$ was calculated as Ib $-1-(2 \times|0.5-\mathrm{p}|)$, where $p$ is the proportion of the 46 accessions that contain the bands (Prevost and Wilkinson, 1999). The PopGene 1.32 software (Yeh, 2000) was used to calculate Nei's genetic diversity and Shannon's information index. A dendrogram was constructed based on Nei's similarity coefficients using the unweighted pair group method with arithmetic average (UPGMA) with the SAHN module of NTSYS-pc 2.2 (Rohlf, 2005). The cophenetic value (COPH) routine and MXCOP modules were used to assess the goodness-of-fit between the cluster analysis and original matrix. The principal coordinated analysis $(\mathrm{PCoA})$ was also conducted to differentiate 
the hybrids and parents in a two-dimensional array of eigenvectors using the DCENTER and EIGEN modules of the NTSYS-pc program.

\section{RESULTS}

\section{Comparative morphology of parental species and their hybrids}

Both parental species $A$. gomosepala and $A$. recurvata var. recurvata had spike-like inflorescence. The spikes of the former were slender and those of the latter were denser. The hybrid plants also had a spike-like inflorescence, but the spikes were morphologically distinct from either of the parental species (Figure 1). The spikes of the hybrids varied in size and were intermediate between both parental species. In addition, the bracts of the hybrids were homogenously scarlet, similar to A. recurvata var. recurvata, while the florets were homogenously blue, similar to A. gomosepala

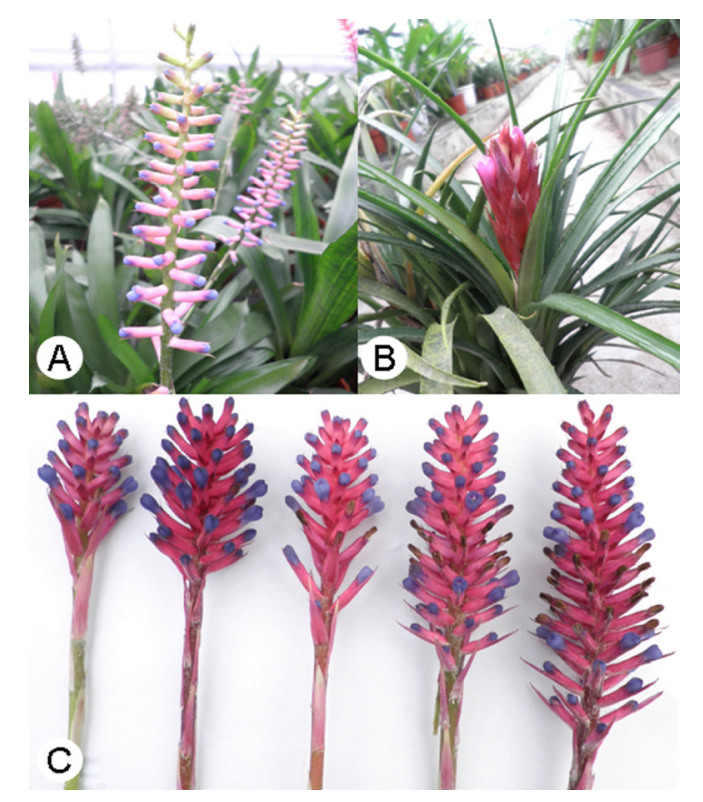

Figure 1. Inflorescence characteristics of Aechmea gomosepala (A), A. recurvata var. recurvata (B), and their interspecific $\mathrm{F}_{1}$ hybrids $(\mathbf{C})$.

\section{SRAP performance}

The result of SRAP analysis is shown in Table 2. For the 40 interspecific hybrids and 2 parental species, the 16 SRAP primer combinations yielded 265 bands, among which 154 $(57.72 \%)$ were polymorphic. The TB generated by each primer combination ranged from 11 to 23 , while the PB ranged from 3 to 17 . The PIC was generally low, with an average of 0.31 and ranging from 0.25 to 0.36 . The $\mathrm{Rp}$ of the informative primer combinations ranged from 1.81 to 12.04 with an average of 6.20. The primer combination Me1Em20, Me3Em21, and Me5Em21 showed the highest $\mathrm{Rp}$ values $(10.38,10.40$, and 12.04, respectively). 
Table 2. Characteristics of examined sequence-related amplified polymorphism primer combinations in the Aechmea hybrids.

\begin{tabular}{|c|c|c|c|c|c|c|c|}
\hline $\mathrm{PC}$ & TB & PB & PPB (\%) & PIC & $\mathrm{Rp}$ & $h$ & $I$ \\
\hline Me21Em1 & 21 & 14 & 66.67 & 0.35 & 10.38 & 0.45 & 0.64 \\
\hline $\mathrm{Me} 21 \mathrm{Em} 1$ & 15 & 5 & 33.33 & 0.31 & 3.05 & 0.40 & 0.58 \\
\hline Me22Em1 & 13 & 9 & 69.23 & 0.32 & 5.86 & 0.41 & 0.60 \\
\hline $\mathrm{Me} 20 \mathrm{Em} 2$ & 21 & 13 & 61.91 & 0.32 & 9.33 & 0.45 & 0.65 \\
\hline $\mathrm{Me} 21 \mathrm{Em} 2$ & 23 & 10 & 43.48 & 0.29 & 6.10 & 0.37 & 0.54 \\
\hline $\mathrm{Me} 22 \mathrm{Em} 2$ & 17 & 16 & 94.12 & 0.29 & 8.14 & 0.30 & 0.47 \\
\hline $\mathrm{Me} 20 \mathrm{Em} 3$ & 17 & 6 & 35.29 & 0.32 & 3.33 & 0.35 & 0.53 \\
\hline $\mathrm{Me} 21 \mathrm{Em} 3$ & 19 & 15 & 78.95 & 0.32 & 10.40 & 0.35 & 0.52 \\
\hline $\mathrm{Me} 22 \mathrm{Em} 3$ & 10 & 4 & 40.00 & 0.30 & 2.52 & 0.28 & 0.44 \\
\hline $\mathrm{Me} 20 \mathrm{Em} 4$ & 14 & 11 & 78.57 & 0.36 & 8.67 & 0.39 & 0.57 \\
\hline $\mathrm{Me} 21 \mathrm{Em} 4$ & 19 & 8 & 42.11 & 0.28 & 4.24 & 0.26 & 0.41 \\
\hline Me20Em5 & 17 & 3 & 17.65 & 0.30 & 1.81 & 0.26 & 0.42 \\
\hline Me21Em5 & 23 & 17 & 73.91 & 0.32 & 12.04 & 0.34 & 0.51 \\
\hline Me20Em6 & 11 & 5 & 45.46 & 0.25 & 2.09 & 0.33 & 0.49 \\
\hline Me21Em6 & 12 & 7 & 58.33 & 0.27 & 3.66 & 0.28 & 0.43 \\
\hline $\mathrm{Me} 22 \mathrm{Em} 6$ & 13 & 11 & 84.62 & 0.33 & 7.62 & 0.36 & 0.54 \\
\hline Average & 16.56 & 9.63 & 57.72 & 0.31 & 6.20 & 0.35 & 0.52 \\
\hline Total & 265 & 154 & - & - & 99.25 & - & - \\
\hline
\end{tabular}

$\mathrm{PC}=$ primer combination; $\mathrm{TB}=$ number of total bands; $\mathrm{PB}=$ number of polymorphic bands; $\mathrm{PPB}=$ percentage of polymorphic bands; PIC = polymorphic information content; $\mathrm{Rp}=$ resolving power; $h=$ Nei's (1973) gene diversity; $I$ = Shannon's information index (Lewontin, 1972).

Of the 154 polymorphic bands, 62 were verified in A. gomosepala, 63 in A. recurvata var. recurvata, 12 in both parental species, and 17 in hybrids but not in parents. A typical amplification profile is shown in Figure 2.

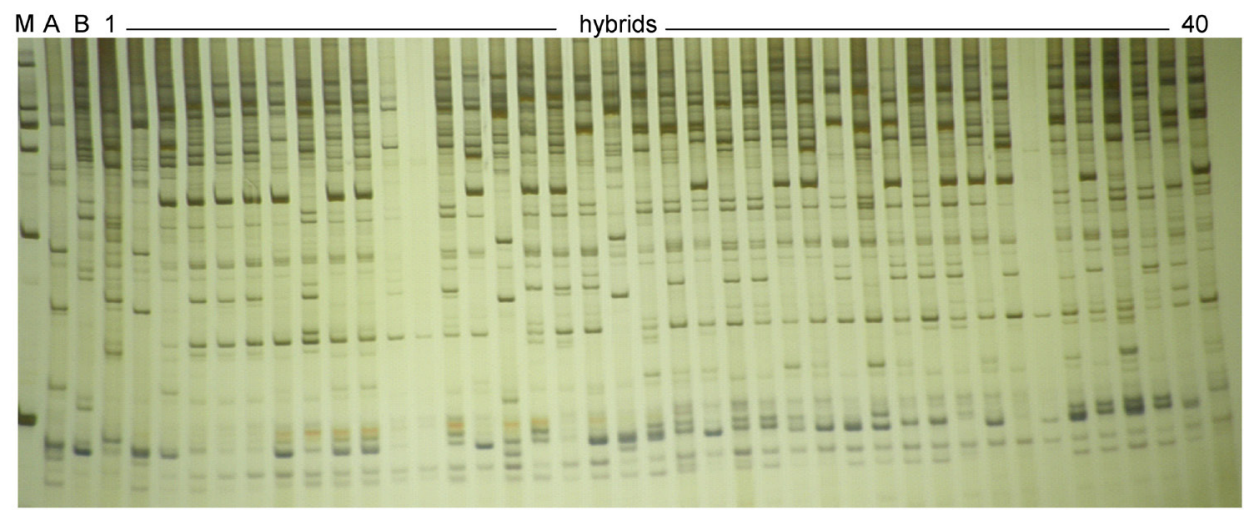

Figure 2. A typical example of sequence-related amplified polymorphism profiles of the 40 interspecific hybrids together with both parental species, obtained from Me21Em5. Lane $M=$ DNA marker ladder; lane A = Aechmea gomosepala; lane $B=A$. recurvata var. recurvata; lanes 1 to $40=$ the interspecific hybrids derived from $A$. gomosepala $\mathrm{x}$. recurvata var. recurvata.

\section{Genetic divergence}

The $h$ ranged from 0.26 to 0.45 with an average of 0.35 , while the $I$ varied from 0.41 to 0.65 with an average of 0.52 (Table 2). The genetic similarity ranged from 0.21 to 0.87 with an average of 0.59 for the whole accessions under study. Additionally, the genetic similarity was normally distributed (Figure 3). 


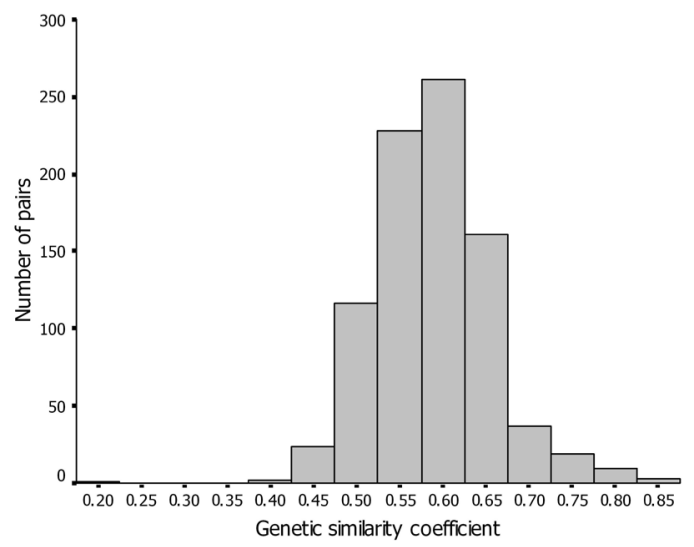

Figure 3. Frequency distributions of sequence-related amplified polymorphism-based genetic similarity estimates for possible pairs of the interspecific hybrids together with both parental species.

The dendrogram generated by UPGMA cluster analysis based on Nei's genetic distance is shown in Figure 4. The cophenetic correlation was estimated to be 0.93, indicating a good fit between the dendrogram and the original distance matrix. According to the dendrogram, the 40 hybrids were grouped into 2 main clusters, and the 2 parental species were clustered into 2 separate clades with a genetic distance of 0.54 . The hybrids were distinguishable from the 2 parental species.

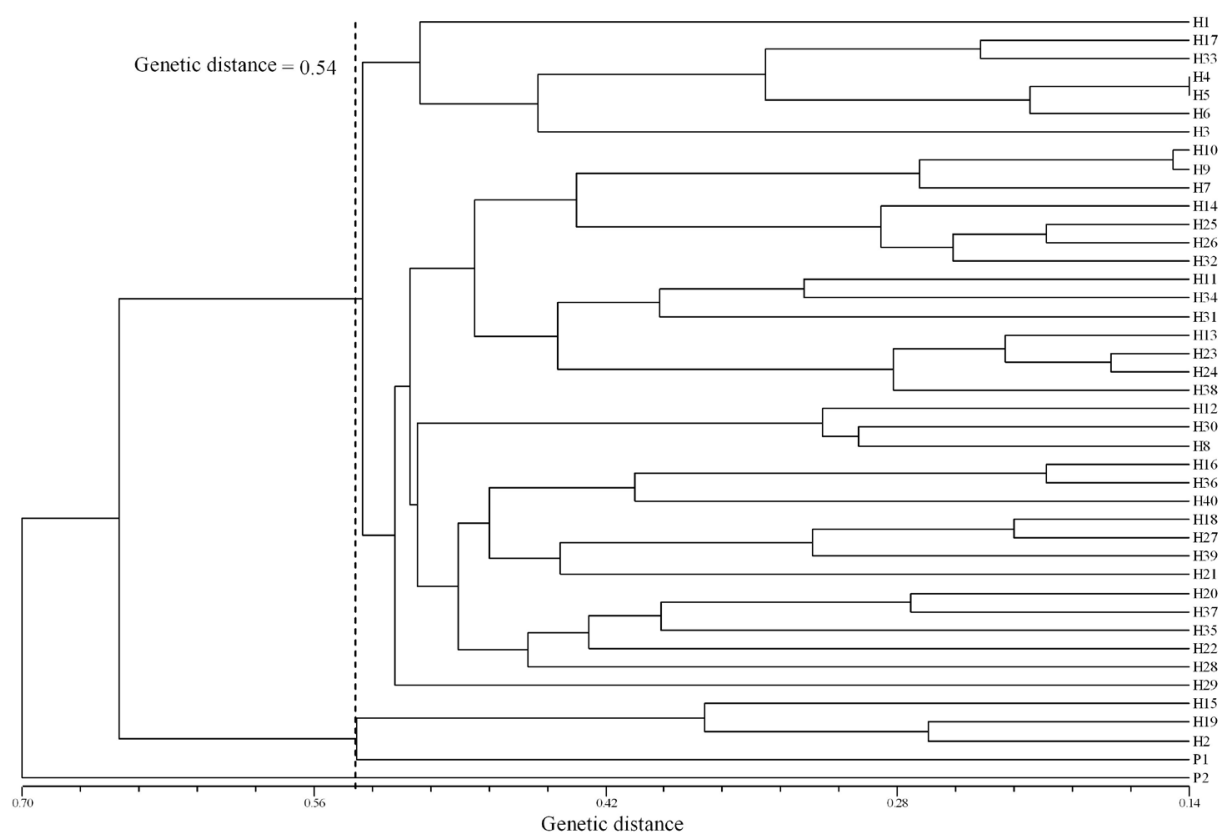

Figure 4. Dendrogram of the interspecific hybrids and both parental species, derived from the UPGMA cluster analysis based on Nei's genetic distance matrix. The codes, P1, P2, and H1 to H40, listed on the right of the dendrogram refer to Aechmea gomosepala, A. recurvata var. recurvata, and the interspecific hybrids, respectively. 
A two-dimensional plot of the PCoA based on the similarity matrix is shown in Figure 5. The first 2 ordinates (PC1 and PC2) accounted for 26.8 and $22.0 \%$, respectively, of the total variation in SRAP markers among the accessions. Generally, the two-dimensional plot demonstrated the expected tendency with an intermediate distribution of the interspecific $\mathrm{F}_{1}$ hybrids relative to parents and that the $\mathrm{F}_{1}$ hybrids dispersed in 2 clusters. As such, the PCoA results generally reinforced the cluster analysis as mentioned above.

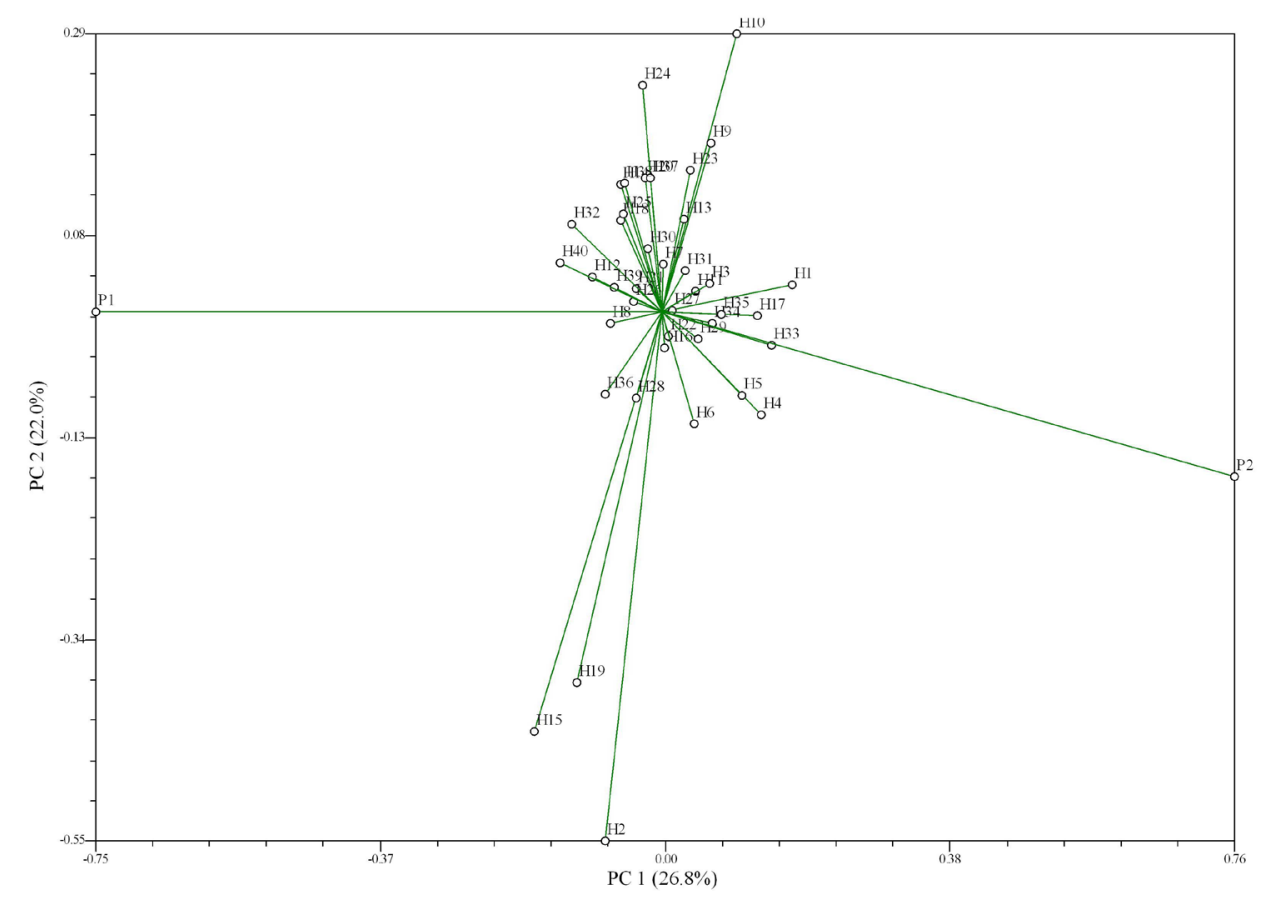

Figure 5. Two-dimensional plot with vectors of principal coordinate analysis (PCoA) of the interspecific hybrids relative to both parental species. The first two principal coordinates (PC1, PC2) accounted for 26.8 and $22.0 \%$ of the molecular variations. The codes, P1, P2 and H1 to H40, refer to Figure 4.

\section{DISCUSSION}

Conventional artificial hybridization and selection techniques have contributed to the development of new cultivars of ornamental crops, including bromeliad. Since bromeliad is a perennial crop that needs about 4 years after seeding to show its typical flower traits, it is time-consuming to identify the hybrids based on phenotype alone. Additionally, the traditional method of hybrid identification based on morphological traits can be influenced by environmental factors and thus lacks the resolution to identify hybrids at an early stage. Advances in DNA fingerprinting techniques have provided diagnostic molecular markers for facilitating the identification of hybrids, including RAPD (Oliveira et al., 2003; Dhillon et al., 2009; Bharathi et al., 2011), ISSR (Bianco et al., 2011), AFLP (Divakaran et al., 2006), and SSR (Saxena et al., 2010; Fu et al., 2011). Recently, many studies have confirmed the high infor- 
mativeness and reproducibility of SRAP markers for identifying and authenticating hybrids in different crops (Liu et al., 2007; Hao et al., 2008; Mishra et al., 2011). In order to provide information on hybridization and selective breeding programs for Aechmea bromeliads, this study investigated the genetic divergence and hybrid nature of interspecific offspring of Aechmea bromeliads using inflorescence characteristics and SRAP profiles. The putative hybrids had inflorescence traits of both parents, suggesting their hybrid nature and the possibility of improving Aechmea bromeliads through artificial hybridization. The PPB of SRAP primer combinations ranged from 17.65 to $94.12 \%$ with an average of $57.72 \%$, and the marker-based genetic similarity ranged from 0.21 to 0.87 with an average of 0.59 . This showed moderate genetic divergence among the inter-specific hybrids investigated.

In this study, most of the primer combinations did not show specific bands in all hybrids despite their presence in the parental species. This demonstrates that the hybrid nature of the offspring could not be determined based on a single locus. However, their hybrid nature could be confirmed by combination of several loci. This result is similar to a previous report that evaluated cabbage hybrids (Liu et al., 2007). Here, $17(\sim 11 \%)$ bands of non-parental origin were observed in some hybrids. Similar results have also been reported in maize (Abdel-Mawgood et al., 2006), Jatropha (Dhillon et al., 2009), and grapes (Bianco et al., 2011). As discussed previously, these hybrid-specific bands may result from recombination, mutation, and rearrangement during the crossing-over phase of meiosis (Dabkevičiene et al., 2008). Similarly, chromosomal crossing-over during meiosis may result in the loss of priming sites, causing markers that are present in parents but not in hybrids (Smith et al., 1996).

The clustering of accessions by UPGMA demonstrates that the hybrids were grouped into 2 subgroups distinct from the 2 parental species. The cluster analysis is more sensitive to closely related individuals, whereas the PCoA is more informative for distances among major groups (Hauser and Crovello, 1982). Therefore, the multivariate approach, PCoA, is often used to complement the cluster analysis (Bianco et al., 2011; Tantasawat et al., 2011). Compared to the UPGMA cluster analysis in this study, the two-dimensional PCoA plot separated the hybrids and both parents into more distinct groups, in agreement with the UPGMA clustering and the morphological analysis. Thus, the present study confirms that SRAP profiling is a practical approach for unequivocally showing the relationship among hybrids and their parents.

In conclusion, this study showed that SRAP profiling was efficient and was able to identify the moderate genetic divergence among the inter-specific hybrids of Aechmea bromeliads. These results suggested that SRAP markers could help identify breeders, characterize the $\mathrm{F}_{1}$ offspring of bromeliads at an early stage, and expedite the genetic improvement of bromeliad cultivars.

\section{ACKNOWLEDGMENTS}

Research supported by the National Natural Science Foundation of China (Grant \#31101570), the Zhejiang Provincial Natural Science Foundation of China (Grant \#Y3110271), the Key Science Technology Specific Projects of Zhejiang Province (Grant \#2009C12095), the Non-profit Technology Research Program of Zhejiang Province (Grant \#2011C22014), the Hangzhou Seeds and Seedlings of Special Project of Zhejiang Province (Grant\#20110332H15), and the Science and Technology Innovation Ability Promoting Project of Zhejiang Academy of Agricultural Sciences (Grant \#2011R25Y01D01). 


\section{REFERENCES}

Abdel-Mawgood AL, Ahmed MMM and Ali BA (2006). Applification of molecular markers for hybrids maize (Zea mays L.) identification. J. Food Agric. Environ. 4: 176-178.

Barfuss MH, Samuel R, Till W and Stuessy TF (2005). Phylogenetic relationships in subfamily Tillandsioideae (Bromeliaceae) based on DNA sequence data from seven plastid regions. Am. J. Bot. 92: 337-351.

Bharathi LK, Vinod, Das AB, Ghosh N, et al. (2011). Cytomorphological and molecular characterization of interspecific F1 hybrid of Momordica dioica Roxb. x Momordica subangulata subsp. Renigera (G. Don) de Wilde. Afr. J. Agric. Res. 6: 2982-2990.

Bianco CL, Farnández JA, Migliaro D, Crinò P, et al. (2011). Identification of F1 hybrids of artichoke by ISSR markers and morphological analysis. Mol. Breed. 27: 157-170.

Boisselier-Dubayle MC, Leblois R, Samadi S, Lambourdiere J, et al. (2010). Genetic structure of the xerophilous bromeliad Pitcairnia geyskesii on inselbergs in French Guiana - a test of the forest refuge hypothesis. Ecography 33: 175-184.

Dabkevičiene G, Paplauskienè V and Pašakinskienè I (2008). Assessment of the agronomic utility of interspecific hybrids Trifolim Pratense L. x T. diffusum Ehrh. and confirmation of their hybridity with ISSR markers. J. Food Agric. Environ. 6: 187-190.

Dhillon RS, Hooda MS, Jattan M, Chawla V, et al. (2009). Development and molecular characterization of interspecific hybrids of Jatropha curcas x J. integerrima. Indian J. Biotechnol. 8: 384-390.

Divakaran M, Babu KN, Ravindran PN and Peter KV (2006). Interspecific hybridization in vanilla and molecular characterization of hybrids and selfed progenies using RAPD and AFLP markers. Sci. Hortic. 108: 414-422.

Fu J, Peng ZJ, Cai XD and Guo WW (2011). Regeneration and molecular characterization of interspecific somatic hybrids between Satsuma mandarin and two seedy sweet oranges for scion improvement. Plant Breed. 130: 287-290.

Han XY, Wang LS, Shu QY, Liu ZA, et al. (2008). Molecular characterization of tree peony germplasm using sequencerelated amplified polymorphism markers. Biochem. Genet. 46: 162-179.

Hao Q, Liu ZA, Shu QY, Zhang R, et al. (2008). Studies on Paeonia cultivars and hybrids identification based on SRAP analysis. Hereditas 145: 38-47.

Hauser LA and Crovello TJ (1982). Numerical analysis of generic relationships in the Thelypodieae (Brossicaceae). Syst. Bot. 7: 249-268.

Izquierdo LY and Pinero D (2000). High genetic diversity in the only known population of Aechmea tuitensis (Bromeliaceae). Aust. J. Bot. 48: 645-650.

Li G and Quiros CF (2001). Sequence-related amplified polymorphism (SRAP), a new marker system based on a simple PCR reaction: its application to mapping and gene tagging in Brassica. Theor. Appl. Genet. 103: 455-461.

Li G, Gao M, Yang B and Quiros CF (2003). Gene for gene alignment between the Brassica and Arabidopsis genomes by direct transcriptome mapping. Theor. Appl. Genet. 107: 168-180.

Liu K and Muse SV (2005). PowerMarker: an integrated analysis environment for genetic marker analysis. Bioinformatics 21: 2128-2129.

Liu L, Liu G and Gong Y (2007). Evaluation of genetic purity of F1 hybrid seeds in cabbage with RAPD, ISSR, SRAP, and SSR markers. Hortscience 42: 724-727.

Mishra MK, Suresh N, Bhat AM, Suryaprakash N, et al. (2011). Genetic molecular analysis of Coffea arabica (Rubiaceae) hybrids using SRAP markers. Rev. Biol. Trop. 59: 607-617.

Murray MG and Thompson WF (1980). Rapid isolation of high molecular weight plant DNA. Nucleic Acids Res. 8: 4321-4325.

Oliveira RP, Aguilar-Vildoso CI and Machado MA (2003). Genetic divergence among hybrids of "Cravo" mandarin with "Pêra" sweet orange. Sci. Agric. 60: 115-118.

Palma-Silva C, Lexer C, Paggi GM, Barbara T, et al. (2009). Range-wide patterns of nuclear and chloroplast DNA diversity in Vriesea gigantea (Bromeliaceae), a neotropical forest species. Heredity 103: 503-512.

Prevost A and Wilkinson MJ (1999). A new system of comparing PCR primers applied to ISSR fingerprinting of potato cultivars. Theor. Appl. Genet. 98: 107-112.

Rohlf FJ (2005). NTSYSpc Numerical Taxonomy and Multivariate Analysis System. Version 2.2. Exeter Software, New York.

Saxena RK, Sexena K and Varshney RK (2010). Application of SSR markers for molecular characterization of hybrid parents and purity assessment of ICPH 2438 hybrid of pigeonpea [Cajanus cajan (L.) Millspaugh]. Mol. Breed. 26: 371-380.

Smith JF, Burke CC and Wagner WL (1996). Interspecific hybridization in natural populations of Cyrtandra (Gesneriaceae) on the Hawaiian Islands: evidences from RAPD markers. Plant Syst. Evol. 200: 61-77.

Sousa GM, Wanderley MGL and Alves M (2008). Inflorescence architecture in Brazilian species of Aechmea subgenus Chevaliera (Bromeliaceae - Bromelioideae). Bot. J. Linn. Soc. 158: 584-592.

Tantasawat P, Trongchuen J, Prajongjai T, Jenweerawat S, et al. (2011). SSR analysis of soybean (Glycine max (L.) Merr.) 
genetic relationship and variety identification in Thailand. Aust. J. Crop Sci. 5: 283-290.

Vervaeke I, Wouters J, Stichelbout L, Londers E, et al. (2003). Inheritance of spineless leaves in Aechmea (Bromeliaceae). Euphytica 134: 47-49.

Yeh F (2000). Popgene 1.32. Available at [http://www.ualberta.ca/ fyeh]. Accessed January 20, 2012.

Zhang F, Chen S, Chen F, Fang W, et al. (2011). SRAP-based mapping and QTL detection for inflorescence-related traits in chrysanthemum (Dendranthema morifolium). Mol. Breed. 27: 11-23. 